\title{
DESARROLLO DE ESTRATEGIAS MEDIANTE CUADRO DE MANDO INTEGRAL PARA LA GESTIÓN DEL TALENTO HUMANO Y EL DESEMPEÑO ADMINISTRATIVO EN LAS EMPRESAS HOTELERAS DE LA PROVINCIA DE SANTA ELENA
}

\section{DEVELOPMENT OF STRATEGIES THROUGH BALANCED SCORECARD FOR THE MANAGEMENT OF HUMAN TALENT AND ADMINISTRATIVE PERFORMANCE IN THE HOTEL COMPANIES OF THE PROVINCE OF SANTA ELENA}

\author{
René Faruk Garzozi Pincay’ Jessenia Quimi Dominguez² Vanessa Suárez Medina \\ Yadira Zambrano Limón
}

\section{Resumen}

\begin{abstract}
El presente trabajo está enfocado en evidenciar los procesos utilizados en las empresas hoteleras para una correcta gestión administrativa realizada por el talento humano que labora dentro de la empresa. Cabe mencionar que en la actualidad las empresas definen como base principal a sus colaboradores. Su importancia radica en el compromiso de alcanzar los objetivos establecidos a mediano y largo plazo, y generar un alto impacto en los procesos, brindando un servicio de calidad dentro de un mercado altamente competitivo, razón por la cual motivan a la empresa a adaptarse a los cambios para gestionar nuevos sistemas que ayuden a potencializar las capacidades del talento humano, con lo cual se crea una ventaja competitiva. El objetivo principal es desarrollar estrategias mediante la aplicación del Cuadro de Mando Integral, que permita mantener una visión estratégica de cada proceso en las empresas hoteleras. Este estudio tiene un enfoque cuantitativo y cualitativo. Los métodos utilizados fueron el inductivo y deductivo, con el fin de analizar las causas y efectos que incidieron en el problema. El tipo de diseño de la investigación se fundamenta en un estudio de carácter exploratorio, descriptivo y bibliográfico, que genera datos relevantes para desarrollar el trabajo investigativo. Se propone una aplicación de cuadros de mando integral en las empresas hoteleras, para establecer estrategias adecuadas en la toma de decisiones. Esta herramienta nos ofrece tener una perspectiva diferente desde el ámbito financiero, clientes, procesos internos, innovación y perspectiva de aprendizaje.
\end{abstract}

\section{Palabras claves}

Talento humano, gestión del talento humano, empresas de servicios, gestión administrativa, Cuadro de Mando Integral.

\section{Abstract}

This work is focused on showing the processes used in hotel companies for a correct administrative management carried out by the human talent that works within the company. It is worth mentioning that at present the companies define their collaborators as their main base. Its importance lies in the commitment to achieve the objectives established in the medium and long term, and to generate a high impact in the processes providing a quality service within a highly competitive market; Reason why they motivate the company to adapt to the changes to manage new systems that help to potentiate the capacities of the human talent, thus creating a competitive advantage. The main objective is to develop strategies through the implementation of the Balanced Scorecard, which allows to maintain a strategic vision of each process in the hotel companies. This study has a quantitative and qualitative approach. The methods used were inductive and deductive; In order to analyze the causes and effects that affected the problem. The type of research design is based on an exploratory, descriptive and bibliographic study that generates relevant data to develop research work. It proposes an application of integral scorecards in the hotel companies by means of which they allow to establish strategies suitable for the decision making. This tool offers us to have a different perspective from the financial field, clients, internal processes, innovation and learning perspective.

\section{Keywords}

Human talent, human talent management, service companies, administrative management, Balance Scorecard.

Universidad Estatal Península de Santa Elena (UPSE), Santa Elena - Ecuador (rgarzozi@upse.edu.ec). Universidad Estatal Península de Santa Elena (UPSE), Santa Elena - Ecuador (jesseniavqd@hotmail.com) Universidad Estatal Península de Santa Elena (UPSE), Santa Elena - Ecuador (vane_stefy14@ahotmail.com) Universidad Estatal Península de Santa Elena (UPSE), Santa Elena - Ecuador (andhyzambrano@gmail.com) 


\section{Introducción}

En el mundo, el ámbito empresarial ha transcendido por causa de la globalización, exigiendo que las multinacionales sean competitivas y estén altamente preparadas para enfrentar situaciones que se requieran de tomas de decisiones rápidas e inmediatas, considerando que dichas decisiones pueden incidir positiva o negativamente en las funciones de la empresa.

La cultura, la política, el ámbito social y la economía son variables que inciden en la correcta gestión del talento humano, y su aplicación tiende a ser muy diferente en cada territorio; es decir, que en empresas del sistema asiático, europeo, norteamericano y latinoamericano, tienen distintas expectativas en relación a los procesos de selección del personal y contratación de su personal, evitando la alta rotación de puestos mediante motivaciones que brinden satisfacción.

La administración del talento humano se encarga de proveer a su equipo de trabajo los recursos necesarios para cumplir con los objetivos establecidos en la organización, con la finalidad de que los colaboradores respondan asertivamente a los distintos escenarios dentro del lugar de trabajo. El principal desafío es gestionar el talento humano con las herramientas apropiadas que permitan proporcionar nuevas expectativas al momento de realizar sus actividades, evidenciando la efectividad en cada etapa del proceso administrativo (Orrego, 2015).

En este tenor $y$, dado que la mayoría de las organizaciones actuales reconocen que la ventaja competitiva proviene más del conocimiento, de las capacidades y las relaciones intangibles creadas por los empleados que, de las inversiones en activos físicos, entonces la aplicación de la estrategia requiere, por lo tanto, que todos los empleados, así como todas las unidades de negocio y de apoyo, estén alineadas y vinculadas a dichas estrategias (Cuahquentzi, Flores, \& Flores, 2017).

En la provincia de Santa Elena las empresas de servicios son muy diversas, por eso la presente investigación se enfoca en aquellas que están direccionadas a brindar servicios a través de los hoteles, captando clientes locales, nacionales e internacionales, aprovechando de alguna manera las riquezas naturales que posee la provincia para generar ingresos y aportar al desarrollo turístico y económico (Palacios, 2017).

El Cuadro de Mando Integral (CMI) es una herramienta estratégica que permite direccionar a la organización, elevando el control y la gestión empresarial para afianzar el posicionamiento dentro de la competencia, haciendo énfasis en cuatro perspectivas, las cuales son: el desempeño financiero, el éxito con los clientes objetivos, los procesos internos y los objetivos de aprendizaje y crecimiento (Norton, 1996).

El objetivo principal es desarrollar estrategias mediante la aplicación de un control de gestión en donde se utiliza el CMI, que permite mantener una visión estratégica de cada proceso administrativo dentro de las empresas hoteleras.

\section{La gestión del talento humano}

La gestión del talento humano tiene como finalidad desarrollar y dar seguimientos al personal para una correcta toma de decisiones, generando así un empoderamiento dentro de la organización más competitivo y dando cumplimiento a los objetivos propuestos (Vallejo, 2016).

Dentro de las empresas hoteleras se consideran varios procesos, entre los cuales mencionamos el administrativo y operativo, como un conjunto de actividades coordinadas que se proyectan bajo ciertas circunstancias, es decir son el conjunto de pasos o etapas necesarias que se deben cumplir de manera planificada (Fulss, 2016). 
La selección del personal es uno de los procesos preponderantes dentro de la gestión administrativa de las empresas hoteleras, la cual permite evaluar de manera sistemática las habilidades y capacidades que puedan cumplir de acuerdo al puesto requerido, por tanto, se plantea un sinnúmero de pruebas que contrastan a los posibles ocupantes y así tomar una decisión más adecuada (Guerrero Reyes \& Galindo Alvarado, 2014).

Por tanto, el personal debe cumplir con las actividades de acuerdo a los conocimientos adquiridos, lo que permite capacidad en la toma de decisiones de manera autónoma, generando así un factor diferenciador dentro del mercado (Villegas, 2015), mientras que el comportamiento organizacional es la conducta que tienen los trabajadores dentro de la empresa, en donde presentan diferentes cambios, que inciden en la aplicación de técnicas adecuadas para una buena gestión del clima organizacional (Durive, 2015).

Se definen como objetivos a la representación de los resultados que se desea lograr dentro de procesos consecutivos. Para alcanzarlos se ejecuta un sinnúmero de estrategias que también se plantean en función de la visión de la organización (Oscco, 2015). El establecimiento de políticas orienta a los trabajadores a desenvolverse de manera adecuada en el medio, respetando los planteamientos y directrices instituidas (Milian, 2015).

La gestión del talento humano implica motivar al personal, aplicando un plan motivacional utilizado como una herramienta para alcanzar un excelente desempeño laboral, que satisfaga las necesidades y anhelos de los trabajadores y genere sentido de pertenencia con la empresa (Torres-Hernández, y otros, 2014), permitiendo que los trabajadores participen en la toma de decisiones, con el compromiso de lograr los resultados deseados, lo que se convierte en un importante factor motivador (Simón, 2006).
Los incentivos salariales son estímulos orientados a mejorar el rendimiento laboral e inciden en la calidad y productividad de la organización (Garcillán, 2017). Pero en una empresa no es muy relevante brindar solo motivaciones económicas, sino ayudar al colaborador a sentirse satisfecho con los beneficios que la empresa le pueda brindar, sea uno de ellos el ascenso a un nivel jerárquico más alto, lo que implica desarrollarse profesionalmente.

Consecuentemente, la capacitación es considerada como una herramienta que ayuda al personal a desarrollar nuevos aprendizaje y conocimientos, y se define como la adquisición de conocimientos técnicos, teóricos y prácticos que van a contribuir al desarrollo del individuo en el desempeño de una actividad. (America, 2015). Un programa de capacitación es de suma importancia para la gestión del talento humano, porque a través de esta se obtiene un capital humano preparado y comprometido a conseguir el éxito de la empresa (Guerrero, 2015).

Habilidad es la capacidad actual de la persona en determinada actividad o comportamiento. La habilidad se adquiere a partir de una aptitud preexistente, mediante la práctica o el ejercicio (Chiavenato, 2011). Mientras que los conocimientos son los aspectos de puesta en práctica del nivel de instrucción académica y la capacitación que posee el personal.

En cuanto a las actitudes, estas engloban un conjunto de creencias, todas ellas relacionadas entre sí, que permiten alcanzar y superar metas, es decir son reacciones que cada empleado tiene frente a cualquier situación en el ámbito laboral, que le permite llegar a la excelencia de un trabajo (Carreño \& Cruz, 2012).

\section{El desempeño administrativo}

La administración es el arte del diseño de los sistemas organizacionales que facilitan la labor colectiva. Cuando el diseño de la or- 
ganización es coherente y el trabajo se realiza dentro de unas coordenadas claras, la misión, los objetivos, la estructura y los procesos contribuirán con un desempeño administrativo exitoso (Torres Laborde \& Jaramillo Naranjo, 2014). Por lo tanto, la formación profesional en el desarrollo individual cobra una gran importancia, pues las capacidades, competencias y actitudes de los actuales y futuros trabajadores tienen un impacto muy alto dentro de la organizaciones (Jaspe, 2015). Además, la remuneración es el aspecto tangible de acuerdo a la excelencia de la contraprestación de los servicios prestados por el colaborador, para la subsistencia del individuo y su grupo familiar. También depende del desempeño o desenvolvimiento del trabajador (Feijoo, 2016).

Cabe mencionar que las condiciones laborales describen el escenario del puesto de trabajo y el entorno que lo rodea; especifica si es un lugar abierto o cerrado y sobre las características del lugar como la ventilación, el ruido, la temperatura, entre otros (Pulgar-Vidal \& Ríos-Ramos, 2015).

\section{Control de gestión del talento humano}

El control de gestión del talento humano es un proceso utilizado por los directivos, tanto de manera objetiva como subjetiva, para influir en las actuaciones de los otros miembros de la organización. Además, es esencial para poner en práctica las estrategias necesarias para alcanzar con eficiencia y eficacia en los objetivos propuestos. Existen múltiples propuestas en relación con los sistemas de control; todos tienen un elemento en común el cual consiste en el interés para contribuir al mejoramiento de las actuaciones de las organizaciones (García, 2015)

En este caso utilizaremos el Cuadro de Mando Integral, también conocido como CMI. Es un instrumento que permite gestionar los procesos de una organización en la cual se traducen estrategias que debe implementar una empresa, sea pública o privada, que proporciona un adecuado funcionamiento del sistema de gestión y de medición estratégica, en el cual se hace énfasis en el logro que se desea alcanzar, determinando de alguna manera los factores clave del éxito. Tiene como objetivo principal describir las estrategias destinadas a la creación del valor del producto o servicio que se brinda a los clientes (Kaplan, 2014).

Por lo tanto, las empresas hoteleras deberían implementar esta herramienta, porque les permite mantener bajo control aquellos aspectos claves que generan valor, y les ofrece la oportunidad de verificar de manera clara el rendimiento de la empresa, con mediciones sobre la base de indicadores y objetivos alcanzados, desde diferentes perspectivas, como:

- Perspectiva financiera: en este aspecto se considera el planteamiento de parámetros relacionados a la rentabilidad de la empresa.

- Perspectiva del cliente: está direccionada a la satisfacción del cliente, para mantenerlo mediante nuevas propuestas de valor que se deseen brindar.

- Perspectiva de procesos internos: se refiere a que todos los procesos que se ejecutan en la empresa, deben ser de calidad.

- Perspectiva de innovación y capacitación: los empleados son el factor principal de la empresa por lo que se debe desarrollar las habilidades y capacidades de los colaboradores (Jiménez Cachay, 2016).

En cuanto al desarrollo del CMl, esto debe estar direccionado a los procesos que se deseen. De ah\{i que se deba empezar por conocer la situación actual del negocio, estableciendo la descomposición de la organización en tres niveles, los cuales corresponden a: 
- Nivel estratégico: comprendido en misión, visión, valores, competencias y lineamientos estratégicos para dar facilidad a la medición del grado de cumplimiento de los objetivos, siendo parte fundamental del control de gestión.

- Nivel organizativo: se refiere al compromiso por parte de los colaboradores de la empresa para obtener la satisfacción del cliente a través de procesos, procedimientos, funciones y estructura organizativa.

- Nivel de recursos: en este aspecto se debe considerar al recurso económico, tecnológico y recurso humano calificado para generar valor agregado.

Establecidos los objetivos estratégicos, se procede a definir los respectivos indicadores en cada una de las perspectivas, así también las metas que permitan verificar el buen desarrollo de la herramienta estratégica a implementar, es decir la factibilidad del CMI (Palacios Acero, 2018).

\section{Semáforo del cuadro integral}

Luego de la puesta en marcha del CMI, se establecen intervalos de medición para los indicadores, con la asignación de tres colores diferentes:

- Verde: en este color se indica el buen funcionamiento y cumplimiento de los objetivos. Es decir, existe una muy buena gestión, por lo que se consigue que los empleados estén satisfechos con su cargo.

- Amarillo: indica que se requiere atención y se debe buscar las causas de ciertas dificultades. La gestión debe mejorar en la empresa, con la implementación de estrategias adecuadas.
- Rojo: no se logró el objetivo y se debe tomar las medidas necesarias para mejorar la situación. Se ha mal gestionado los procesos y se debe considerar la implementación de nuevas estrategias para el logro efectivo del objetivo (Espinosa, 2015).

\section{Materiales y métodos}

En el presente trabajo se aplicó la investigación de tipo descriptiva, considerando aspectos cualitativos y cuantitativos, con la finalidad de recolectar información verídica y oportuna a través de la encuesta, lo que permitió profundizar el tema en desarrollo.

El instrumento utilizado fue la encuesta, dirigida hacia los gerentes de empresas de servicio de alojamiento como son: hostales, casas de huéspedes, hoteles, hosterías y resorts, de la provincia de Santa Elena, entre los cuales encontramos un total de 170 establecimientos. Sin embargo, la investigación solo se enfoca en el servicio que brindan los hoteles de la provincia, con una muestra de 46 hoteles en los diferentes cantones. Se utilizó el muestreo no probabilístico por conveniencia, para facilitar la accesibilidad y proximidad de la información.

\section{Resultado}

La encuesta fue aplicada a las empresas hoteleras de la provincia de Santa Elena, en donde nos enfocamos en indagar sobre la importancia que tiene la gestión del talento humano en el desempeño administrativo; sin embargo, solo se considera los datos más relevantes de la encuesta. Para una adecuada interpretación de los datos, a continuación se presenta los resultados obtenidos: 
Tabla 1. El perfil de cargo en empresas hoteleras

\begin{tabular}{|c|l|c|c|c|c|}
\hline \multicolumn{5}{|c|}{ ¿La selección del personal debe ir de acuerdo al perfil para el cargo? } \\
\hline \multirow{2}{*}{ Ítems } & \multicolumn{1}{|c|}{ Valoración } & Frecuencia & Porcentaje & Porcentaje válido & $\begin{array}{c}\text { Porcentaje } \\
\text { acumulado }\end{array}$ \\
\hline \multirow{5}{*}{2} & Rara vez & 3 & 6.5 & 6.5 & 6.5 \\
\cline { 2 - 6 } & A veces & 10 & 21.7 & 21.7 & 28.3 \\
\cline { 2 - 6 } & Frecuentemente & 16 & 34.8 & 34.8 & 63.0 \\
\cline { 2 - 6 } & Siempre & 17 & 37.0 & 37.0 & 100.0 \\
\cline { 2 - 6 } & Total & 46 & 100.0 & 100.0 & \\
\hline
\end{tabular}

Figura 1. El perfil de cargo en empresas hoteleras

\section{¿La selección del personal debe ir de acuerdo al perfil para el cargo?}

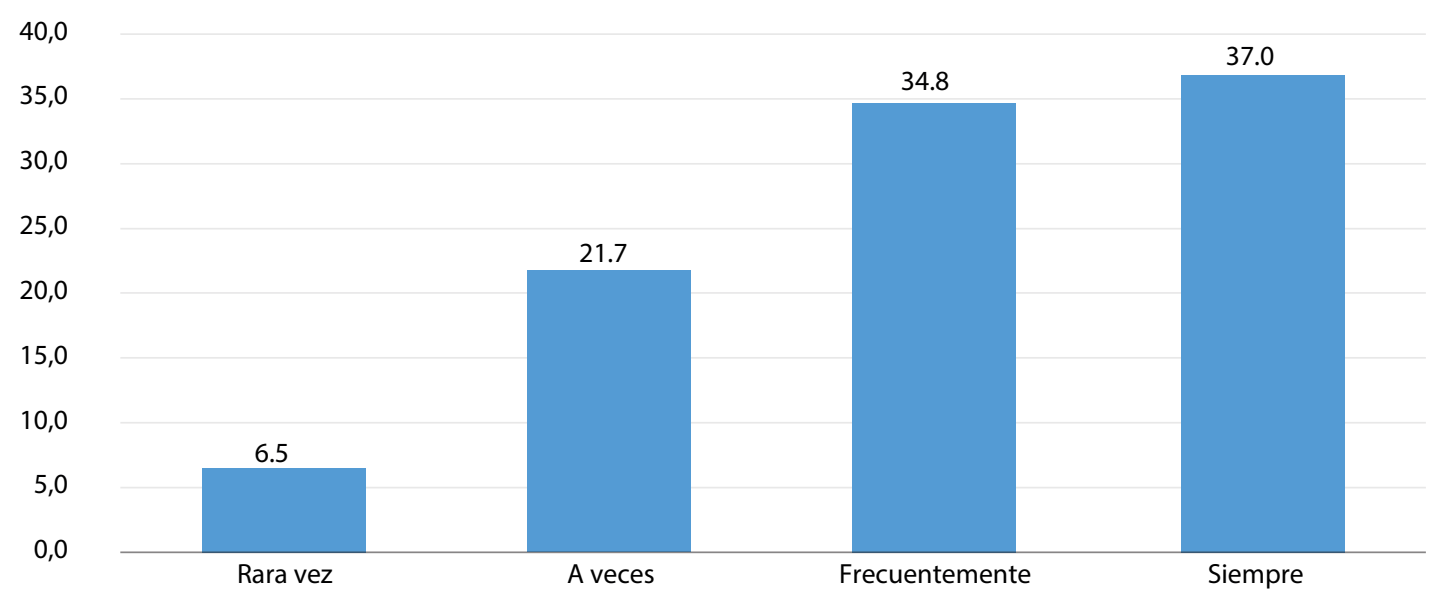

De acuerdo a las encuestas realizadas en la presente investigación, se puede indicar, que un mayor porcentaje de los dueños de es- tablecimientos hoteleros determinan que es de mucha relevancia cumplir un perfil acorde al cargo que se va a desempeñar en la empresa.

Tabla 2. La participación laboral entre compañeros

\begin{tabular}{|c|l|c|c|c|c|}
\hline \multicolumn{6}{|c|}{ ¿Se logra una buena participación laboral con todos sus compañeros de trabajo? } \\
\hline \multirow{2}{*}{ Ítems } & \multicolumn{1}{|c|}{ Valoración } & Frecuencia & Porcentaje & $\begin{array}{c}\text { Porcentaje } \\
\text { válido }\end{array}$ & $\begin{array}{c}\text { Porcentaje } \\
\text { acumulado }\end{array}$ \\
\hline \multirow{4}{*}{5} & Nunca & 4 & 8.7 & 8.7 & 8.7 \\
\cline { 2 - 6 } & Rara vez & 7 & 15.2 & 15.2 & 23.9 \\
\cline { 2 - 7 } & A veces & 16 & 34.8 & 34.8 & 58.7 \\
\cline { 2 - 6 } & Frecuentemente & 14 & 30.4 & 30.4 & 89.1 \\
\cline { 2 - 6 } & Siempre & 5 & 10.9 & 10.9 & 100 \\
\cline { 2 - 6 } & Total & 46 & 100.0 & 100.0 & \\
\hline
\end{tabular}


Figura 2. La participación laboral entre compañeros

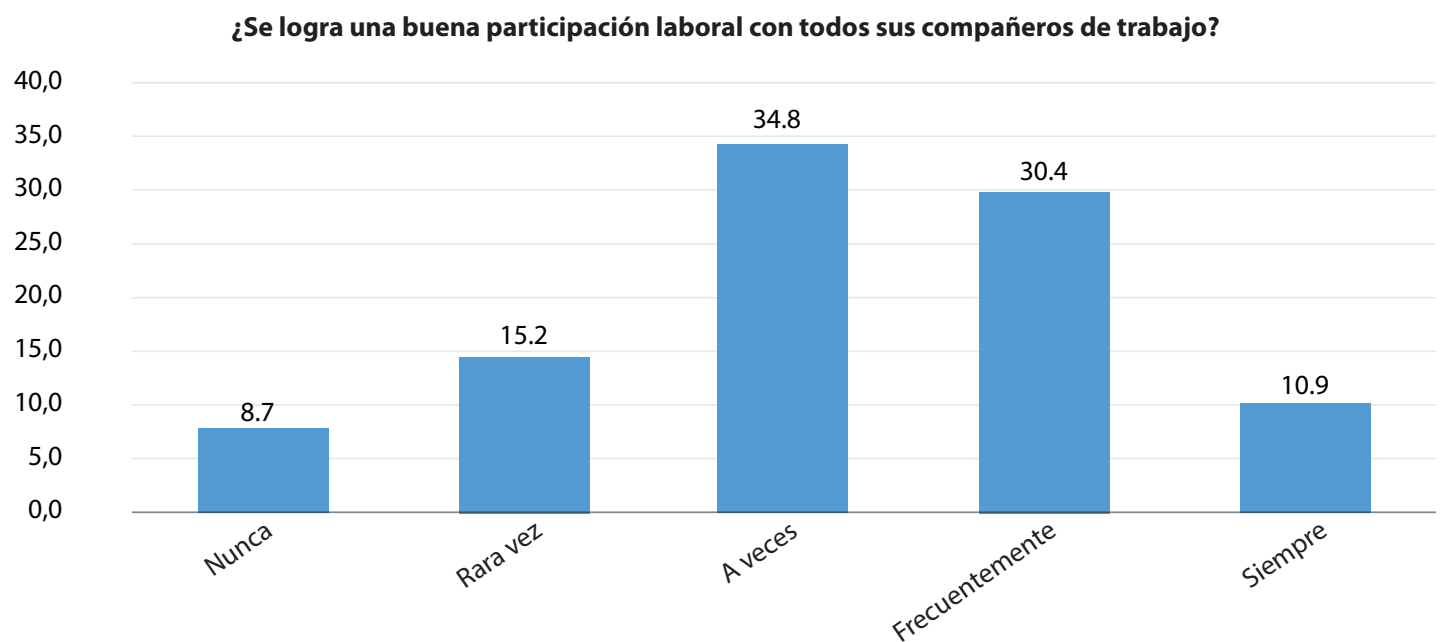

Mediante el estudio realizado se concluye que un mayor porcentaje de los trabajadores sí está conforme con la participación laboral que comparte con sus compañeros de trabajo, y solo una menor parte dice no poseer una buena participación en el área laboral.

Tabla 3. Capacitaciones y nivel de competencia

\begin{tabular}{|c|l|c|c|c|c|}
\hline \multicolumn{5}{|c|}{ ¿Los planes o programas de capacitación ayudan a mejorar el nivel de competencia? } \\
\hline \multirow{2}{*}{ Ítems } & \multicolumn{1}{|c|}{ Valoración } & Frecuencia & Porcentaje & $\begin{array}{c}\text { Porcentaje } \\
\text { válido }\end{array}$ & $\begin{array}{c}\text { Porcentaje } \\
\text { acumulado }\end{array}$ \\
\hline \multirow{3}{*}{8} & Frecuentemente & 13 & 28.3 & 28.3 & 28.3 \\
\cline { 2 - 6 } & Siempre & 33 & 71.7 & 71.7 & 100.0 \\
\cline { 2 - 6 } & Total & 46 & 100.0 & 100.0 & \\
\hline
\end{tabular}

Figura 3. Capacitaciones y nivel de competencia

¿Los planes o programas de capacitación ayudan a mejorar el nivel de competición?

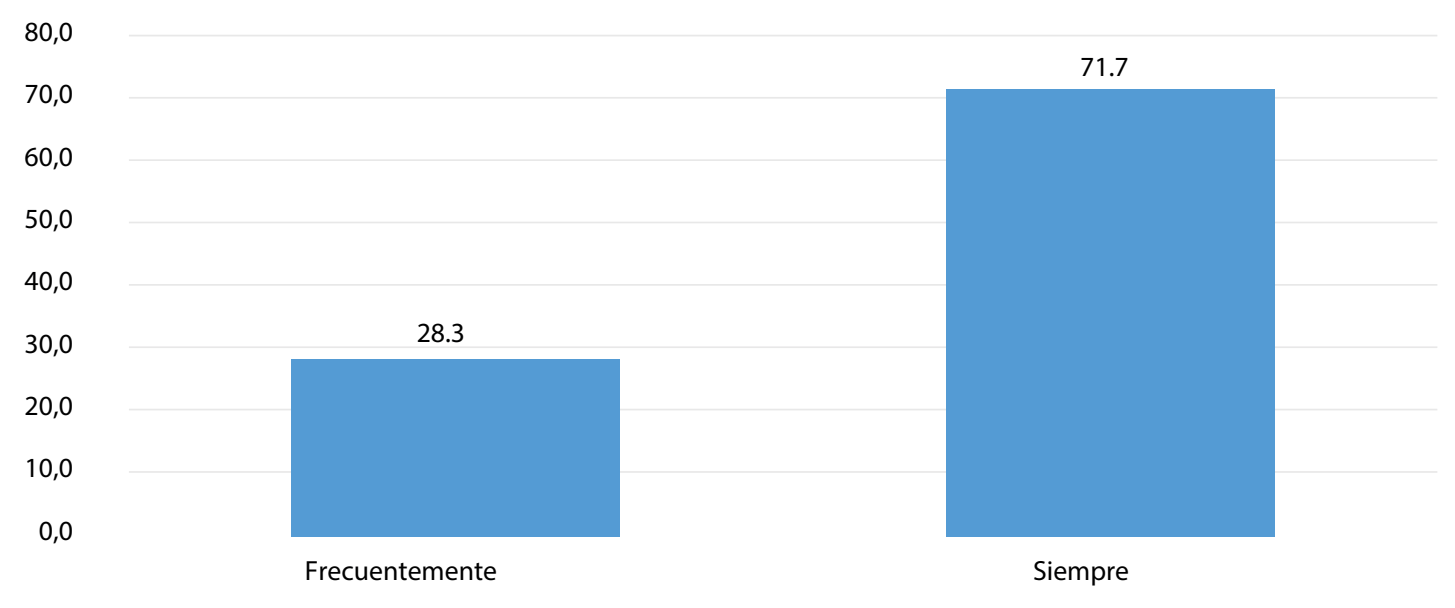


Es adecuado determinar que la mayor parte de los encuestados están de acuerdo en que las capacitaciones ayudan a mejorar el nivel de productividad ante la competen- cia, al dar un servicio de calidad y calidez a sus clientes; mientras que a una pequeña cantidad no le es relevante recibir capacitaciones de manera periódica.

Tabla 4. La interacción del talento humano

\begin{tabular}{|c|l|c|c|c|c|}
\hline \multicolumn{6}{|c|}{ ¿Se desarrolla la interacción de la gestión del talento humano con la estrategia } \\
y los procesos dentro de la empresa?
\end{tabular}

Figura 4. La interacción del talento humano

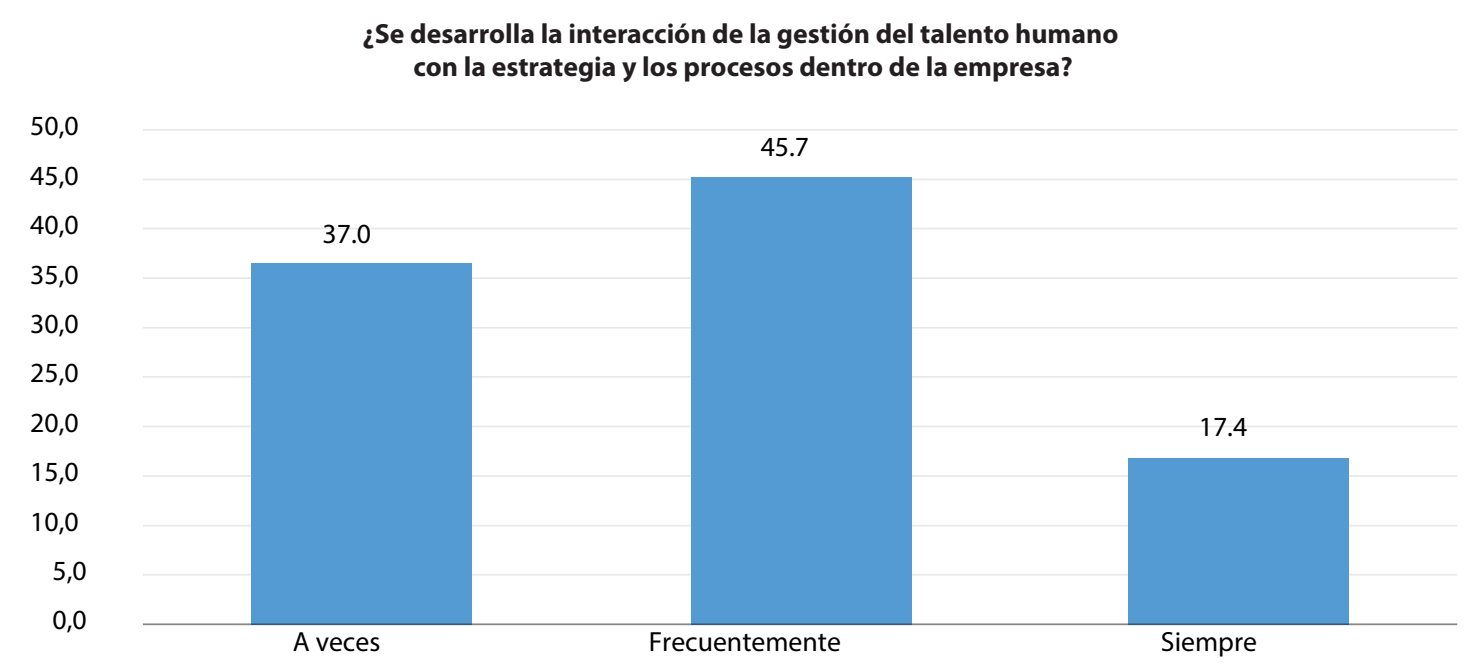

Una mayor parte de la población encuestada determina que, con frecuencia, se desarrollan interacciones, las cuales incluyen diferentes estrategias y procesos realizados dentro de la empresa, seguida de otra parte que dice que se realiza de manera periódica.

\section{Conclusión}

La importancia de gestionar el talento humano recae en que esto es el factor principal para cumplir con los objetivos establecidos en la empresa, por lo que se debe buscar y gestionar las estrategias que permitan desarrollar de manera adecuada el personal, mejorando el desempeño que ayude a la adaptación fácil a los cambios constantes de nuestro entorno. El talento humano es el único que después de una capacitación y entrenamiento puede generar mayor productividad con eficiencia y eficacia, utilizando adecuadamente los recursos necesarios y así generar una atención más fluida a los usuarios.

El CMI es una herramienta que ayuda a las empresas a establecer estrategias adecuadas desde las perspectivas del nivel financiero, los clientes, los procesos internos y el aprendizaje. Los procesos de selección, 
capacitación y evaluación de desempeño están direccionados a mejorar de forma efectiva el desempeño del trabajador, lo cual se direcciona a obtener una buena calidad en sus procesos, dando cavidad a reducir costos e incrementar las ganancias de las empresas hoteleras.

\section{Aporte de la investigación}

El modelo estratégico de $\mathrm{CMI}$ en estos tipos de empresas hoteleras permitirá mejorar eficientemente los procesos administrativos que efectúa el talento humano, facilitando la comunicación y gestión, comprometiendo al personal a mejorar a través de capacitaciones que incidan en lograr resultados exitosos para la empresa. El administrador del talento humano en este tipo de empresas debe promover la creatividad, innovación, el trabajo en equipo, la efectividad y la capacitación de todo el personal, en forma permanente y constante, considerando los cambios del entorno.

El modelo de CMI en las empresas hoteleras se sugiere con la siguiente estructura, que garantizará el buen funcionamiento de los procesos a través del planteamiento de indicadores y objetivos:

6. Antecedentes de la empresa.

7. Realizar el análisis FODA con su respectiva matriz estratégica.
8. Establecer la misión, visión, valores y objetivos.

9. Desarrollo de estrategias a través de las distintas perspectivas, tales como: Financiero: rendimiento sobre la inversión, crecimiento de los ingresos (costo de servicio por habitación), tarifa promedio de la habitación.

Cliente: Aumentar la cuota de mercado, mejorar la atención del cliente (tiempo, calidad y atributos diferenciadores), retención de los clientes, satisfacción del cliente y rentabilidad del cliente, establecer precios acordes al mercado y segmento donde se maneja la empresa.

Proceso interno: habitaciones de acuerdo a las normas; crear relaciones ganar-ganar con proveedores, definir las funciones y responsabilidades de todos los miembros de la empresa. Aprendizaje y capacitación: logro de competencias, compromiso de recursos humanos, perfeccionar el talento humano sobre la base de la capacitación constante para que sea competitivo.

De acuerdo a la información desarrollada, se procede a establecer el siguiente $\mathrm{CMI}$, donde se describe los objetivos estratégicos, indicadores y estrategias:

CMI para las empresas hoteleras de la provincia de Santa Elena

\begin{tabular}{|c|c|c|c|}
\hline Perspectiva & $\begin{array}{l}\text { Objetivos } \\
\text { estratégicos }\end{array}$ & Indicadores & Estrategias \\
\hline \multirow{5}{*}{ Financiera } & \multirow{5}{*}{$\begin{array}{l}\text { Mejorar } \\
\text { la rentabilidad } \\
\text { del negocio }\end{array}$} & Número de reservaciones & $\begin{array}{l}\text { Incrementar los niveles de } \\
\text { ingreso }\end{array}$ \\
\hline & & Nivel de ingresos & Optimizar costos y gastos \\
\hline & & $\begin{array}{l}\text { Número de metas asignadas/Incre- } \\
\text { mento de ventas }\end{array}$ & Fortalecer el prestigio \\
\hline & & $\begin{array}{l}\mathrm{N}^{\circ} \text { de reservaciones perdidas } / \mathrm{N}^{\circ} \\
\text { total de reservaciones }\end{array}$ & $\begin{array}{l}\text { Elevar el nivel de satisfacción de } \\
\text { los clientes }\end{array}$ \\
\hline & & $\mathrm{N}^{\circ}$ de promociones mensuales & Fidelizar clientes rentables \\
\hline
\end{tabular}




\begin{tabular}{|c|c|c|c|}
\hline \multirow{3}{*}{$\begin{array}{l}\text { Procesos } \\
\text { internos }\end{array}$} & \multirow{3}{*}{$\begin{array}{l}\text { Mantener la } \\
\text { calidad de los } \\
\text { procesos }\end{array}$} & $\begin{array}{l}\text { Horas de capacitación impartida/Ho- } \\
\text { ras de capacitación programadas }\end{array}$ & Mejorar calidad de servicio \\
\hline & & $\mathrm{N}^{\circ}$ de publicidad & $\begin{array}{l}\text { Incrementar la participación en la } \\
\text { promoción de producto turístico }\end{array}$ \\
\hline & & $\begin{array}{l}\mathrm{N}^{\circ} \text { de vallas publicitarias } / \mathrm{N}^{\circ} \text { clientes } \\
\text { que visitan }\end{array}$ & $\begin{array}{l}\text { Mejorar la imagen del producto } \\
\text { turístico }\end{array}$ \\
\hline \multirow{4}{*}{$\begin{array}{l}\text { Aprendizaje y } \\
\text { capacitación }\end{array}$} & \multirow{4}{*}{$\begin{array}{l}\text { Optimizar el } \\
\text { capital humano }\end{array}$} & $\begin{array}{l}\mathrm{N}^{\circ} \text { de capacitaciones/total de } \\
\text { colaboradores }\end{array}$ & Fortalecer valores institucionales \\
\hline & & $\begin{array}{l}\text { Encuestas sobre clima laboral/ total } \\
\text { de instrumentos de medición de } \\
\text { clima organizacional }\end{array}$ & Mejorar clima organizacional \\
\hline & & $\mathrm{N}^{\circ}$ de capacitaciones programadas & $\begin{array}{l}\text { Direccionar el entrenamiento y la } \\
\text { capacitación }\end{array}$ \\
\hline & & $\begin{array}{l}\text { Horas hombre trabajadas } / \mathrm{N}^{\circ} \text { de } \\
\text { trabajadores }\end{array}$ & $\begin{array}{l}\text { Aumentar motivación del } \\
\text { personal }\end{array}$ \\
\hline
\end{tabular}

Adaptado de: Robert Kaplan \& David Norton, 1996.

\section{Plan de capacitación}

Capacitar al personal, inculcando la filosofía empresarial y el buen servicio al cliente, y ayudando a promover el uso eficiente de los recursos y cumplir con las normativas requeridas para las empresas hoteleras.

\begin{tabular}{|c|c|c|c|c|c|c|c|}
\hline \multirow{2}{*}{ RECURSOS } & \multirow{2}{*}{ costo } & \multirow{2}{*}{ ACTIV. } & \multirow{2}{*}{ PLAZO } & \multicolumn{3}{|c|}{ CRONOGRAMA 2018} & \multirow{2}{*}{ RESPONSABLE } \\
\hline & & & & ENE. & FEB. & MAR. & \\
\hline \multirow{4}{*}{$\begin{array}{l}\text { Recursos } \\
\text { Humanos } \\
\text { Administrado- } \\
\text { res Capacitado- } \\
\text { res Materiales } \\
\text { Equipos de } \\
\text { oficinas compu- } \\
\text { tación Materia- } \\
\text { les de oficinas } \\
\text { Muebles de } \\
\text { oficinas Equipo } \\
\text { de Audio }\end{array}$} & \multirow{4}{*}{860} & $\begin{array}{l}\text { Taller de sentido } \\
\text { de pertenencia } \\
\text { a la empresa }\end{array}$ & 3 & & & & \multirow{4}{*}{ Capacitadores } \\
\hline & & $\begin{array}{l}\text { Conferencia de } \\
\text { valores y ética } \\
\text { profesional }\end{array}$ & 2 & & & & \\
\hline & & $\begin{array}{l}\text { Servicio y aten- } \\
\text { ción al cliente. }\end{array}$ & 2 & & & & \\
\hline & & $\begin{array}{l}\text { Capacitación } \\
\text { de filosofía } \\
\text { empresarial }\end{array}$ & 4 & & & & \\
\hline
\end{tabular}

Fuente: Plan motivacional anual.

Elaborado por: Autores.

\section{Referencias bibliográficas}

America. (2015). América Latina: Importancia de la capacitación virtual para el sector logístico. Obtenido de America Learning
\& Media: http://www.americalearningmedia.com/edicion-015/173-noticias/2091america-latina-importancia-de-la-capacitacion-virtual-para-el-sector-logistico

Carreño, Á. B., \& Cruz, M. D. (2012). Motivos, actitudes y estrategias de aprendizaje: Apren- 
dizaje motivado en alumnos universitarios. Profesorado. Revista de currículum y formación de profesorado, 16 (1), 125-142.

Chiavenato, I. (2011). Recursos Humanos El Capital Humano De Las Organizaciones (Novena Edición ed.). México: MC GRAW HILL.

Cuahquentzi, M. P., Flores, E. H., \& Flores, G. H. (2017). El impacto del capital humano en el sector hotelero en el Estado de Tlaxcala, desde la perspectiva del Balanced Scorecard. Repositorio de la Red Internacional de Investigadores en Competitividad, 11, 1751-1766.

Durive, L. C. (2015). Importancia de la cultura organizacional para el desarrollo del sistema de gestión de la calidad (Vol. Num.5). Cuba: Ciencia en su PC. Obtenido de http://repositoriodigital.academica.mx/ jspui/ handle/987654321/475822

Espinosa, R. J. (2015). Control de gestión: Facultad de Ciencias Económicas e Informática, Universidad de Matanzas. Cuba: GEOCUBA Matanzas.

Feijoo, J. L. (2016). El Talento Humano en Hotelería y Turismo. (U. Editor, Ed.)

Fulss, D. C. (2016). Conceptos generales sobre enfoque de procesos de negocios. (CAIGG, Ed.) Chile: Ministerio Secretaría General de la Presidencia.

García, A. R. (2015). El control de gestion del talento humano: conceptos y enfoques. Universidad \& Empresas, 29(21:6-7).

Garcillán, M. D. (2017). ¿Cuáles son los mejores incentivos laborales para tus empleados? España.

Guerrero Reyes, C., \& Galindo Alvarado, F. (2014). Administración 2. Serie Integral por Competencias. México: Grupo Editorial Patria.

Guerrero, J. (2015). Programa de capacitación en inteligencia emocional con técnicas cognitivo-conductuales para los directivos de educación. Recuperado de: http://wWW. archivos_revista/419, 20, 138-154.

Jaspe, E. E. (2015). Estrategias Metodólogicas para el Desarrollo Curricular de la Especialización de la Gestión del Talento Humano de las Organizaciones. (J. I. Rodriguez, Ed.) Editorial La Habana.

Jiménez Cachay, G. I. (2016). Cuadro de mando integral y su apoyo al desarrollo estrate- gico de la municipalidad distrital de pataz. Trujillo -Perú: Universidad Privada Antenor Orrego - UPAO.

Kaplan, R. (2014). El cuadro de mando integral: The balanced scorecard (Tercera Edición ed.). (D. P. Norton, Ed.) Harvard Business Press.

Milian, A. M. (2015). Enfoque sistémico del clima organizacional. Revista Cubana, 14(1).

Norton, R. K. (1996). The Balanced ScoreCard: Translating strategy Into action. Boston: Harvard Business School Press.

Orrego, B. E. (2015). Caracterización del Talento Humano en EMpresas del Valle del Cauca. Revista Internacional Administración \& Finanzas, 8(26: 1-2).

Oscco, H. (2015). Gestión del talento humano y su relación con el desempeño laboral del personal de la municipalidad distrital de Pacucha Andahuaylas-Apurímac. Perú:: Andahuaylas.

Palacios Acero, L. C. (2018). Dirección Estratégica. ECOE Ediciones. Segunda Edición.

Palacios, J. (Septiembre de 2017). Analisis de los atributos de la oferta de los atributos de la oferta de servicios turisticos en la provincia de Santa Elena. Ecorfan, 3(60: 18).

Pulgar-Vidal, L. H., \& Ríos-Ramos, F. (2015). Metodología para Implantar la Estrategia: Diseño Organizacional de la Empresa. (F. R. Ramos, Ed.) Lima: Universidad Peruana de Ciencias Aplicadas (UPC).

Simón, R. (2006). Las claves de las organizaciones de alto rendimiento. España: Ediciones Deusto.

Torres Laborde, J. L., \& Jaramillo Naranjo, O. L. (2014). Diseño y análisis de puestos de trabajo: Herramienta para la gestion del Talento Humano. (O. L. Jaramillo, Ed.) Barranquilla: Editorial Universidad del Norte.

Torres-Hernández, Z., Torres-Martínez, H., RojasPantoja, J., Ramírez-Flores, J., RodríguezPerego, N., \& Meza-Olvera, E. (2014). Administración de proyectos (Grupo Editrial Patria ed.). México: Grupo Editorial Patria.

Vallejo. (2016). Gestión del talento humano, Aval ESPOCH. Riobamba: La Caracola.

Villegas, A. M. (2015). Terminología pedagógica especifica al enfoque por competencia. Bélgica: Universidad Católica de Lovaina (UCL). 